\title{
Relation between Small Functions with Differential Polynomials Generated by Solutions of Linear Differential Equations
}

\author{
Zhigang Huang \\ School of Mathematics and Physics, Suzhou University of Science and Technology, Suzhou 215009, China
}

Correspondence should be addressed to Zhigang Huang, alexehuang@sina.com

Received 11 October 2011; Accepted 11 January 2012

Academic Editor: Wolfgang Ruess

Copyright (C) 2012 Zhigang Huang. This is an open access article distributed under the Creative Commons Attribution License, which permits unrestricted use, distribution, and reproduction in any medium, provided the original work is properly cited.

This paper is devoted to studying the growth of solutions of second-order nonhomogeneous linear differential equation with meromorphic coefficients. We also discuss the relationship between small functions and differential polynomials $L(f)=d_{2} f^{\prime \prime}+d_{1} f^{\prime}+d_{0} f$ generated by solutions of the above equation, where $d_{0}(z), d_{1}(z)$, and $d_{2}(z)$ are entire functions that are not all equal to zero.

\section{Introduction and Main Results}

A function $f(z)$ is called meromorphic if it is nonconstant and analytic in the complex plane $\mathbb{C}$ except at possible isolated poles. If no poles occur, then $f(z)$ reduces to an entire function. Throughout this paper, we assume that the reader is familiar with the fundamental results and the standard notations of the Nevanlinna's value distribution theory of meromorphic functions, for reference see [1]. In addition, we use notations $\sigma(f)$ and $\lambda(f)$ to denote the order and the exponent of convergence of zero sequence and $\bar{\lambda}(f)$ to denote the sequence of distinct zeros of $f(z)$, respectively. A meromorphic function $\psi(z)$ is called a small function with respect to $f(z)$ if $T(r, \psi)=o(T(r, f))$ as $r \rightarrow \infty$, possibly outside of a set of $r$ with finite measure, where $T(r, f)$ is the Nevanlinna characteristic function of $f(z)$.

For the second-order linear differential equation

$$
f^{\prime \prime}+e^{-z} f^{\prime}+B(z) f=0
$$


where $B(z)$ is an entire function of finite order, it is well known that each solution $f$ of (1.1) is an entire function, and that if $f_{1}$ and $f_{2}$ are any two linearly independent solutions of (1.1), then at least one of $f_{1}, f_{2}$ must have infinite order, see [2, pages 167-168].

Thus, a natural question is the following: what condition on $B(z)$ will guarantee that every solution $f \not \equiv 0$ of (1.1) has infinite order? Many researchers have studied the question, for the details see [3, page 291]. For the case that $B(z)$ is a transcendental entire function, Gundersen [4] proved that if $\sigma(B) \neq 1$, then every solution $f \neq \equiv 0$ of (1.1) has infinite order. In 2002 , Chen considered the problem and proved the following result which is an improvement of Gundersen's result.

Theorem A (see [3]). Let $a, b$ be nonzero complex numbers satisfying $a b \neq 0$ and $\arg a \neq \arg b$ or $a=c b(0<c<1)$, and let $A_{j}(z) \not \equiv 0(j=1,2)$ be entire functions with $\sigma\left(A_{j}\right)<1$, then every solution $f(\not \equiv)$ of the equation

$$
f^{\prime \prime}+A_{1}(z) e^{a z} f^{\prime}+A_{0}(z) e^{b z} f=0
$$

has infinite order.

Some further results on (1.2) were obtained for several cases. Chen [3] got the same conclusion when $a=c b(c>1)$, and Chen and Shon [5] investigated the more general equations with meromorphic coefficients. Under the same assumption of Theorem $A$, if $A_{1}(z)$ and $A_{0}(z)$ are meromorphic functions with $\sigma\left(A_{j}\right)<1(j=0,1)$, then there is the same conclusion with Theorem $A$. In 2008, Wang and Laine [6] extended Theorem A to nonhomogeneous second-order linear differential equations.

Theorem B (see [6]). Let $A_{j}(z) \not \equiv 0(j=0,1)$ and $F \not \equiv 0$ be entire functions with $\max \left\{\sigma\left(A_{j}\right),(j=\right.$ $0,1), \sigma(F)\}<1$, and let $a, b$ be complex constants that satisfy $a b \neq 0$ and $a \neq b$, then every solution $f$ of differential equation

$$
f^{\prime \prime}+A_{1}(z) e^{a z} f^{\prime}+A_{0}(z) e^{b z} f=F
$$

is of infinite order.

Remark 1.1. Belaïdi and El Farissi [7] also proved Theorem B and got $\bar{\lambda}(f)=\lambda(f)=\sigma(f)=\infty$. We note that (2.21) in [7] cannot be deduced by following their proof. Indeed, as $r \rightarrow \infty$, $|f(z)|>1$ holds just for the points $z$ satisfying $|f(z)|=M(r, f)$, not for all $z$. However, the difficulty can be got over by using Lemmas 2.5 and 2.6 in [8], and the method can be used in our proof of the following Theorem 1.2.

Since the beginning of the last four decades, a substantial number of research papers have been written to describe the fixed points of general transcendental functions. However, there are few studies on the fixed points of solutions of the general differential equations. In 2000, Chen [9] first studied the problems on the fixed points of solutions of secondorder linear differential equations with entire coefficients. Since then, many results on fixed points of solutions of differential equations with entire coefficients were obtained, see [1012]. In 2006, Chen and Shon [13] further studied the relation between small functions and solutions or differential polynomials of solutions of differential equations and obtained the following. 
Theorem C. Let $A_{j}(z) \not \equiv 0(j=0,1)$ be entire functions with $\sigma\left(A_{j}\right)<1$, and let $a, b$ be complex constants such that $a b \neq 0$ and $\arg a \neq \arg b$ or $a=c b(0<c<1)$. If $\psi(z) \not \equiv 0$ is an entire function with finite order, then every solution $f \not \equiv 0$ of $(1.2)$ satisfies $\bar{\lambda}(f-\psi)=\bar{\lambda}\left(f^{\prime}-\psi\right)=\bar{\lambda}\left(f^{\prime \prime}-\psi\right)=\infty$. Furthermore, let $d_{0}(z), d_{1}(z)$, and $d_{2}(z)$ be polynomials that are not all equal to zero, and let $L(f)=$ $d_{2} f^{\prime \prime}+d_{1} f^{\prime}+d_{0} f$. If the order of $\psi$ is less than 1 , then $\bar{\lambda}(L(f)-\psi)=\infty$.

Belaïdi and El Farissi [7] also studied the relation between small functions and some differential polynomials generated by solutions of the second-order nonhomogeneous linear differential equation (1.3). They obtained the following.

Theorem D. Let $A_{j}(z) \not \equiv 0(j=0,1)$ and $F \not \equiv 0$ be entire functions with $\max \left\{\sigma\left(A_{j}\right) \quad(j=\right.$ $0,1), \sigma(F)\}<1$, and let $a, b$ be complex constants that satisfy $a b \neq 0$ and $\arg a \neq \arg b$ or $a=$ $c b(0<c<1)$. Let $d_{0}(z), d_{1}(z), d_{2}(z)$ be entire functions that are not all equal to zero with $\sigma\left(d_{j}\right)<1(j=0,1,2)$, and let $\psi(z)$ be an entire function with finite order. If $f$ is a solution of (1.3), then the differential polynomial $L(f)=d_{2} f^{\prime \prime}+d_{1} f^{\prime}+d_{0} f$ satisfies $\bar{\lambda}(L(f)-\psi)=\infty$.

The main purpose of this paper is to study the growth and the oscillation of solutions of second-order linear differential equation with meromorphic coefficients. Also, we will investigate the relation between small functions and differential polynomials generated by solutions of the above equation. Our results can be stated as follows.

Theorem 1.2. Let $A_{j}(z) \not \equiv 0(j=0,1)$ and $F(z)$ be meromorphic functions with $\max \{\sigma(F)$, $\left.\sigma\left(A_{j}\right)\right\}<n$, and let $P(z)=a_{n} z^{n}+\cdots+a_{0}, Q(z)=b_{n} z^{n}+\cdots+b_{0}$ be polynomials with degree $n(n \geq 1)$, where $a_{i}, b_{i}(i=0,1, \ldots, n), a_{n} b_{n} \neq 0$ are complex constants such that $\arg a_{n} \neq \arg b_{n}$ or $a_{n}=c b_{n}(0<c<1)$, then every meromorphic solution $f \not \equiv 0$ of the equation

$$
f^{\prime \prime}+A_{1} e^{P(z)} f^{\prime}+A_{0} e^{Q(z)} f=F
$$

has infinite order and satisfies

$$
\bar{\lambda}(f)=\lambda(f)=\sigma(f)=\infty
$$

Theorem 1.3. Under the assumption of Theorem 1.2, and let $d_{0}(z), d_{1}(z), d_{2}(z)$ be meromorphic functions that are not all equal to zero with $\sigma\left(d_{j}\right)<1(j=0,1,2)$, and let $\psi(z)$ be a meromorphic function with finite order, if $f \not \equiv 0$ is a meromorphic solution of (1.4), then the differential polynomial $L(f)=d_{2} f^{\prime \prime}+d_{1} f^{\prime}+d_{0} f$ satisfies $\bar{\lambda}(L(f)-\psi)=\infty$.

Remark 1.4. Clearly, the method used in linear differential equations with entire coefficients cannot deal with the case of meromorphic coefficients. In addition, the proof of the results in $[7,13]$ relies heavily on the idea of Lemma 5 in [13] or Lemma 2.5 in [7]. However, it seems too complicated to deal with our cases. We will use an important result in uniqueness theory of meromorphic functions, that is Lemma 2.5 , to prove our theorems.

\section{Preliminary Lemmas}

In order to prove our theorems, we need the following lemmas. 
Lemma 2.1 (see [14]). Let $w(z)$ be a transcendental meromorphic function with $\sigma(f)=\sigma<\infty$. Let $\Gamma=\left\{\left(k_{1}, j_{1}\right), \ldots,\left(k_{m}, j_{m}\right)\right\}$ be a finite set of distinct pairs of integers satisfying $k_{i}>j_{i} \geq 0$ for $i=1,2, \ldots, m$. Also let $\epsilon>0$ be a given constant, then there exists a set $E_{1} \subset(1, \infty)$ that has finite logarithmic measure, such that for all $z$ satisfying $z \notin E \cup[0,1]$ and for all $(k, j) \in \Gamma$, one has

$$
\frac{\left|w^{(k)}(z)\right|}{\left|w^{(j)}(z)\right|} \leq|z|^{(k-j)(\sigma-1+\varepsilon)} \text {. }
$$

Now we introduced a notation, see [15] and [8, Lemma 2.3]. Let $P(z)=(\alpha+\beta i) z^{n}+\cdots$ is a nonconstant polynomial, and $\alpha, \beta$ is real constants. For $\theta \in[0,2 \pi)$, set $\delta(P(z), \theta)=\alpha \cos n \theta-$ $\beta \sin n \theta$.

Lemma 2.2 (see [15]). Let $P(z)$ be a nonconstant polynomial of degree $n$. Let $w(z)$ be a meromorphic function, not identically zero, of order less than $n$, and set $g(z)=w(z) e^{P(z)}$. Then for any given $\varepsilon>0$ there exists a zero measure set $H_{1} \subset[0,2 \pi)$ such that if $\theta \in \theta \in[0,2 \pi) \backslash\left(H_{1} \cup H_{2}\right)$, then for $|z|>r(\theta)$,

(1) if $\delta(P, \theta)<0$, then $\exp \left((1+\epsilon) \delta(P, \theta) r^{n}\right) \leq\left|g\left(r e^{i \theta}\right)\right| \leq \exp \left((1-\epsilon) \delta(P, \theta) r^{n}\right)$,

(2) if $\delta(P, \theta)>0$, then $\exp \left((1-\epsilon) \delta(P, \theta) r^{n}\right) \leq\left|g\left(r e^{i \theta}\right)\right| \leq \exp \left((1+\epsilon) \delta(P, \theta) r^{n}\right)$, where $H_{2}=\{\theta: \delta(P, \theta)=0,0 \leq \theta<2 \pi\}$ is a finite set.

Lemma 2.3 (see [8, Lemma 2.5]). Let $f(z)$ be an entire function, and suppose that

$$
G(z):=\frac{\log ^{+}\left|f^{(k)}\right|}{|z|^{\rho}}
$$

is unbounded on some ray $\arg z=\theta$ with constant $\rho>0$, then there exists an infinite sequence of points $z_{n}=r_{n} e^{i \theta}(n=1,2, \ldots)$, where $r_{n} \rightarrow \infty$, such that $G\left(z_{n}\right) \rightarrow \infty$ and

$$
\frac{\left|f^{(j)}\left(z_{n}\right)\right|}{\left|f^{(k)}\left(z_{n}\right)\right|} \leq \frac{1}{(k-j) !}(1+o(1)) r_{n}^{k-j}, \quad j=0, \ldots, k-1,
$$

as $n \rightarrow \infty$.

Lemma 2.4 (see [16]). Let $A_{0}, \ldots, A_{k-1}, F \neq \equiv 0$ be finite-order meromorphic functions. If $f$ is an infinite-order meromorphic solution of the equation

$$
f^{(k)}+A_{k-1} f^{(k-1)}+\cdots+A_{0} f=F,
$$

then $f$ satisfies $\lambda(f)=\bar{\lambda}(f)=\sigma(f)=\infty$.

Lemma 2.5 (see [17, page 79]). Suppose that $f_{1}(z), f_{2}(z), \ldots, f_{n}(z)(n \geq 2)$ are meromorphic functions and $g_{1}(z), g_{2}(z), \ldots, g_{n}(z)$ are entire functions satisfying the following conditions:

(1) $\sum_{j=1}^{n} f_{j}(z) e^{g_{j}(z)} \equiv 0$,

(2) $g_{j}(z)-g_{k}(z)$ are not constants for $1 \leq j<k \leq n$, 
(3) for $1 \leq j \leq n, 1 \leq h<k \leq n, T\left(r, f_{j}\right)=o\left\{T\left(r, e^{g_{h}-g_{k}}\right)\right\}(r \rightarrow \infty, r \notin E)$, where E has a finite measure.

then $f_{j}(z) \equiv 0(j=1,2, \ldots, n)$.

Lemma 2.6 (see [8, Lemma 2.6]). Let $f(z)$ be a an entire function of order $\sigma(f)=\sigma<\infty$. Suppose that there exists a set $E \subset[0,2 \pi)$ which has linear measure zero, such that $\log ^{+}\left|f\left(r e^{i \theta}\right)\right| \leq M r^{\rho}$ for any ray $\arg z=\theta \in[0,2 \pi) \backslash E$, where $M$ is a positive constant depending on $\theta$, while $\rho$ is a positive constant independent of $\theta$. Then $\sigma(f) \leq \rho$.

Lemma 2.7. Under the assumption of Theorem 1.2, and let $f$ be a meromorphic solution of (1.4). Set $w=f^{\prime \prime}+A_{1} e^{P(z)} f^{\prime}+A_{0} e^{Q(z)} f$. If $f \not \equiv 0$ is of finite order, then $\sigma(w) \geq n$.

Proof. Suppose the contrary that $\sigma(w)<n$, we will deduce a contradiction.

First, if $f(z) \equiv C \neq 0$, then $w=C A_{0} e^{Q(z)}$. Clearly, $\sigma(w)=n$, this is a contradiction.

Now suppose that $f \not \equiv C$. If $\sigma(f)<n$, then

$$
f^{\prime \prime}+A_{1} e^{P(z)} f^{\prime}+A_{0} e^{Q(z)} f-w=0
$$

By Lemma 2.5, we have $A_{0} \equiv 0$, and this is a contradiction. Hence, $\sigma(f) \geq n$.

Since $f$ is a meromorphic solution of (1.4), we know that the poles of $f$ can occur only at the poles of $A_{j}(j=0,1)$ and $F$. Let $f=g(z) / d(z)$, where $d(z)$ is the canonical product formed with the nonzero poles of $f(z)$, with $\sigma(d) \leq \max \left\{\sigma(F), \sigma\left(A_{j}\right), j=0,1\right\}<n$, and $g$ is an entire function with $n \leq \sigma(g)=\sigma(f)=\sigma \leq \infty$. Substituting $f=g / d$ into (2.5), by some calculation we can get

$$
d w=g^{\prime \prime}+g^{\prime}\left[A_{1} e^{P(z)}-2\left(\frac{d^{\prime}}{d}\right)\right]+g\left[A_{0} e^{Q(z)}-A_{1} e^{P(z)} \frac{d^{\prime}}{d}+2\left(\frac{d^{\prime}}{d}\right)^{2}-\frac{d^{\prime \prime}}{d}\right]
$$

Now, we rewrite (2.6) into

$$
\frac{d w}{g}-\frac{g^{\prime \prime}}{g}-\left[A_{1} e^{P(z)}-2\left(\frac{d^{\prime}}{d}\right)\right] \frac{g^{\prime}}{g}=\left[A_{0} e^{Q(z)}-A_{1} e^{P(z)} \frac{d^{\prime}}{d}+2\left(\frac{d^{\prime}}{d}\right)^{2}-\frac{d^{\prime \prime}}{d}\right] .
$$

Set $\max \left\{\sigma(w), \sigma\left(A_{j}\right), j=0,1\right\}=\beta<n$. By Lemma 2.1, for any given $\varepsilon(0<\varepsilon<1-\beta)$, there exists a set $E_{2} \in[0,2 \pi)$ which has linear measure zero, such that if $\theta \in[0,2 \pi) \backslash E_{2}$, then there is a constant $R_{1}=r_{1}(\theta)>1$ such that for all $z$ satisfying $\arg z=\theta$ and $|z| \geq R_{1}$, we have

$$
\frac{\left|g^{(i)}(z)\right|}{|g(z)|} \leq|z|^{2(\sigma-1+\varepsilon)}, \quad \frac{\left|d^{(i)}(z)\right|}{|d(z)|} \leq|z|^{2(\beta-1+\varepsilon)}, \quad i=1,2
$$


Case 1. Suppose that $a_{n}=c b_{n}(0<c<1)$, then by Lemmas 2.1 and 2.2, there exists a ray $\arg z=\theta \in[0,2 \pi) \backslash E_{2} \cup H_{1} \cup H_{2}, H_{1}$ and $H_{2}$ being defined in Lemma 2.2, such that $\delta(P, \theta)=$ $c \delta(Q, \theta)>0$, and for the above $\varepsilon$ and sufficiently $r$,

$$
\begin{aligned}
\left|A_{0} e^{Q(z)}-A_{1} e^{P(z)} \frac{d^{\prime}}{d}+2\left(\frac{d^{\prime}}{d}\right)^{2}-\frac{d^{\prime \prime}}{d}\right| & \geq\left|A_{0} e^{Q(z)}\right|-\left|A_{1} e^{P(z)}\right|\left|\frac{d^{\prime}}{d}\right|-\left|2\left(\frac{d^{\prime}}{d}\right)^{2}\right|-\left|\frac{d^{\prime \prime}}{d}\right| \\
& \geq \frac{1}{2} \exp \left\{(1-\varepsilon) \delta(Q, \theta) r^{n}\right\} .
\end{aligned}
$$

Also, by Lemmas 2.1 and 2.2, we have

$$
\left|A_{1} e^{P(z)}-2\left(\frac{d^{\prime}}{d}\right)\right| \leq\left|A_{1} e^{P(z)}\right|+\left|\frac{2 d^{\prime}}{d}\right| \leq M \exp \left\{(1+\varepsilon) c \delta(Q, \theta) r^{n}\right\}
$$

where $M$ is a constant.

Now we claim that

$$
\frac{\log ^{+}|g(z)|}{|z|^{\beta+\varepsilon}}
$$

is bounded on the ray $\arg z=\theta$. Otherwise, by Lemma 2.3, there exists a sequence of points $z_{m}=r_{m} e^{i \theta}$, such that $r_{m} \rightarrow \infty$

$$
\frac{\log ^{+}\left|g\left(z_{m}\right)\right|}{r_{m}^{\beta+\varepsilon}} \longrightarrow \infty
$$

From (2.12) and the definition of order, we see that

$$
\left|\frac{d\left(z_{m}\right) w\left(z_{m}\right)}{g\left(z_{m}\right)}\right| \rightarrow 0
$$

for $m$ is large enough. By (2.7), (2.8), (2.9), (2.10), and (2.13), we get

$$
\begin{aligned}
\frac{1}{2} \exp \left\{(1-\varepsilon) \delta(Q, \theta) r_{m}^{n}\right\} & \leq\left|A_{0} e^{Q(z)}-A_{1} e^{P(z)} \frac{d^{\prime}}{d}+2\left(\frac{d^{\prime}}{d}\right)^{2}-\frac{d^{\prime \prime}}{d}\right| \\
& \leq\left|\frac{d w}{g}\right|+\left|\left(A_{1} e^{P(z)}-2\left(\frac{d^{\prime}}{d}\right)\right) \frac{g^{\prime}}{g}\right|+\left|\frac{g^{\prime \prime}}{g}\right| \\
& \leq M_{1} \exp \left\{(1+\varepsilon) c \delta(Q, \theta) r_{m}^{n}\right\},
\end{aligned}
$$


where $M_{1}$ is a constant. Clearly, we can choose $\varepsilon$ such that $0<\varepsilon<(1-c) /(1+c)$. Then by (2.14), we can obtain a contradiction. Therefore,

$$
\frac{\log ^{+}|g(z)|}{|z|^{\beta+\varepsilon}}
$$

is bounded, and we have $|g(z)| \leq M \exp \left\{r^{\beta+\varepsilon}\right\}$ on the ray $\arg z=\theta$.

Case 2. Suppose that $\arg a_{n} \neq \arg b_{n}$. By Lemma 2.2, there exists a ray $\arg z=\theta \in[0,2 \pi) \backslash E_{2} \cup$ $H_{1} \cup H_{2}$, where $E_{2}, H_{1}$, and $H_{2}$ are defined, respectively, as in Case 1 , such that

$$
\delta(Q, \theta)>0, \quad \delta(P, \theta)<0
$$

Then, for any given $\varepsilon(0<\varepsilon<n-\beta)$, by Lemma 2.2 and (2.7), we have, for sufficiently large $|z|=r$,

$$
\begin{aligned}
\left|A_{0} e^{Q(z)}-A_{1} e^{P(z)} \frac{d^{\prime}}{d}+2\left(\frac{d^{\prime}}{d}\right)^{2}-\frac{d^{\prime \prime}}{d}\right| & \geq\left|A_{0} e^{Q(z)}\right|-\left|A_{1} e^{P(z)}\right|\left|\frac{d^{\prime}}{d}\right|-\left|2\left(\frac{d^{\prime}}{d}\right)^{2}\right|-\left|\frac{d^{\prime \prime}}{d}\right| \\
& \geq \frac{1}{2} \exp \left\{(1-\varepsilon) \delta(Q, \theta) r^{n}\right\}, \\
\left|A_{1} e^{P(z)}-2\left(\frac{d^{\prime}}{d}\right)\right| & \leq\left|A_{1} e^{P(z)}\right|+2\left|\left(\frac{d^{\prime}}{d}\right)\right| \\
& \leq \exp \{\beta+\varepsilon\} \exp \left\{(1-\varepsilon) \delta(P, \theta) r^{n}\right\}+r^{2(\beta-1+\varepsilon)}
\end{aligned}
$$

As in Case 1, we prove that

$$
\frac{\log ^{+}|g(z)|}{|z|^{\beta+\varepsilon}}
$$

is bounded on the ray $\arg z=\theta$. Otherwise, similarly as in Case 1, there exists a sequence of points $z_{m}=r_{m} e^{i \theta}$, such that $r_{m} \rightarrow \infty$,

$$
\frac{\log ^{+}\left|g\left(z_{m}\right)\right|}{r_{m}^{\beta+\varepsilon}} \longrightarrow \infty
$$

Further, we have

$$
\left|\frac{d\left(z_{m}\right) w\left(z_{m}\right)}{g\left(z_{m}\right)}\right| \longrightarrow 0
$$

for $m$ is large enough. 
By (2.7), (2.8), (2.17), (2.18), and (2.21), we get

$$
\begin{aligned}
\frac{1}{2} \exp \left\{(1-\varepsilon) \delta(Q, \theta) r_{m}^{n}\right\} & \leq\left|A_{0} e^{Q(z)}-A_{1} e^{P(z)} \frac{d^{\prime}}{d}+2\left(\frac{d^{\prime}}{d}\right)^{2}-\frac{d^{\prime \prime}}{d}\right| \\
& \leq\left|\frac{d w}{g}\right|+\left|\left(A_{1} e^{P(z)}-2\left(\frac{d^{\prime}}{d}\right)\right) \frac{g^{\prime}}{g}\right|+\left|\frac{g^{\prime \prime}}{g}\right| \\
& \leq \exp \left\{(1-\varepsilon) \delta(P, \theta) r_{m}^{n}\right\}+r_{m}^{2(\beta-1+\varepsilon)}
\end{aligned}
$$

Since $\delta(Q, \theta)>0$ and $\delta(P, \theta)<0$, we obtain a contradiction. So

$$
\frac{\log ^{+}|g(z)|}{|z|^{\beta+\varepsilon}}
$$

is bounded, and we have

$$
|g(z)| \leq M \exp \left\{r^{\beta+\varepsilon}\right\}
$$

on the ray $\arg z=\theta$.

Combining Cases 1 and 2, for any given ray $\arg z=\theta \in[0,2 \pi) \backslash E, E$ of linear measure zero, we have (2.24) on the ray $\arg z=\theta$, provided that $r$ is sufficiently large. Thus by Lemma 2.6, we get $\sigma(g) \leq \beta+\varepsilon<n$, which is a contradiction. Then $\sigma(w) \geq n$.

Lemma 2.8. Under the assumption of Theorem 1.3, let $f(z)$ be an infinite-order meromorphic solution of $(1.4)$, then $\sigma(L(f))=\infty$.

Proof. Suppose that $f(z)$ is a meromorphic solution of (1.4), then by Theorem 1.2, we have $\sigma(f)=\infty$.

Now suppose that $d_{2} \not \equiv 0$. Substituting $f^{\prime \prime}=F-A_{1} e^{P(z)} f^{\prime}-A_{0} e^{Q(z)}$ into $L(f)$, we have

$$
L(f)-d_{2} F=\left(d_{1}-d_{2} A_{1} e^{P(z)}\right) f^{\prime}+\left(d_{0}-d_{2} A_{0} e^{Q(z)}\right) f
$$
obtain

Differentiating both sides of (2.25), and replacing $f^{\prime \prime}$ with $f^{\prime \prime}=F-A_{1} e^{P} f^{\prime}-A_{0} e^{Q} f$, we

$$
\begin{aligned}
L(f)^{\prime} & -\left(d_{2} F\right)^{\prime}-\left(d_{1}-d_{2} A_{1} e^{P}\right) F \\
= & {\left[d_{2} A_{1}^{2} e^{2 P}-\left(\left(d_{2} A_{1}\right)^{\prime}+P^{\prime} d_{2} A_{1}+d_{1} A_{1}\right) e^{P}-d_{2} A_{0} e^{Q}+d_{0}+d_{1}^{\prime}\right] f^{\prime} } \\
& +\left[d_{2} A_{0} A_{1} e^{P+Q}-\left(\left(d_{2} A_{0}\right)^{\prime}+Q^{\prime} d_{2} A_{0}+d_{1} A_{0}\right) e^{Q}+d_{0}^{\prime}\right] f .
\end{aligned}
$$


Set

$$
\begin{gathered}
\alpha_{1}=d_{1}-d_{2} A_{1} e^{P}, \quad \alpha_{0}=d_{0}-d_{2} A_{0} e^{Q}, \\
\beta_{1}=d_{2} A_{1}^{2} e^{2 P}-\left(\left(d_{2} A_{1}\right)^{\prime}+P^{\prime} d_{2} A_{1}+d_{1} A_{1}\right) e^{P}-d_{2} A_{0} e^{Q}+d_{0}+d_{1}^{\prime}, \\
\beta_{0}=d_{2} A_{0} A_{1} e^{P+Q}-\left(\left(d_{2} A_{0}\right)^{\prime}+Q^{\prime} d_{2} A_{0}+d_{1} A_{0}\right) e^{Q}+d_{0}^{\prime} .
\end{gathered}
$$

Then we rewrite (2.25) and (2.26) into

$$
\begin{gathered}
\alpha_{1} f^{\prime}+\alpha_{0} f=L(f)-d_{2} F \\
\beta_{1} f^{\prime}+\beta_{0} f=L(f)^{\prime}-\left(d_{2} F\right)^{\prime}-\left(d_{1}-d_{2} A_{1} e^{P}\right) F .
\end{gathered}
$$

Set

$$
\begin{aligned}
h=\alpha_{1} \beta_{0} & -\alpha_{0} \beta_{1} \\
= & {\left[d_{1}-d_{2} A_{1} e^{P}\right]\left[d_{2} A_{0} A_{1} e^{P+Q}-\left(\left(d_{2} A_{0}\right)^{\prime}+Q^{\prime} d_{2} A_{0}+d_{1} A_{0}\right) e^{Q}+d_{0}^{\prime}\right] } \\
& -\left[d_{0}-d_{2} A_{0} e^{Q}\right]\left[d_{2} A_{1}^{2} e^{2 P}-\left(\left(d_{2} A_{1}\right)^{\prime}+P^{\prime} d_{2} A_{1}+d_{1} A_{1}\right) e^{P}\right. \\
& \left.-d_{2} A_{0} e^{Q}+d_{0}+d_{1}^{\prime}\right] \\
= & h_{0}+h_{P}(z) e^{P}+h_{P+Q} e^{P+Q}+h_{Q} e^{Q}+h_{2 P} e^{2 P}-d_{2}^{2} A_{0}^{2} e^{2 Q},
\end{aligned}
$$

where $h_{i}(z)(i \in \Lambda=\{0, P, Q, P+Q, 2 P\})$ are meromorphic functions formed by $A_{0}, A_{1}, d_{0}$, and $d_{1}$ and their derivatives, with order less than $n$, and $\Lambda$ is a index set. Since any one of $\{P, Q, P+Q, 2 P\}$ is not equal to $2 Q$, then by Lemma 2.5 , we have $d_{2}^{2} A_{0}^{2} \equiv 0$. This is a contradiction. Thus, $h \neq \equiv$.

By (2.28), we get

$$
f=\frac{1}{h}\left(\alpha_{1}\left(L(f)^{\prime}-\left(d_{2} F\right)^{\prime}-\alpha_{1} F\right)-\beta_{1}\left(L(f)-d_{2} F\right)\right)
$$

If $\sigma(L(f))<\infty$, then by $(2.30)$ we have $\sigma(f)<\infty$. Clearly, it is a contradiction. Hence, $\sigma(L(f))=\infty$.

Suppose that $d_{2} \equiv 0, d_{1} \not \equiv 0$ or $d_{2}=d_{1} \equiv 0$, and $d_{0} \not \equiv 0$, then by similar discussion as above, we can get the same conclusion.

\section{Proof of Theorem 1.2}

Let $f \not \equiv 0$ be a meromorphic solution of (1.4). Conversely, suppose that $\sigma(f)<\infty$. By Lemma 2.7, we have $n \leq \sigma(w)=\sigma(F)<n$. This is a contradiction. By Lemma 2.4, $f$ satisfies $\bar{\lambda}(f)=\lambda(f)=\sigma(f)=\infty$. 


\section{Proof of Theorem 1.3}

Suppose that $\arg a_{n} \neq \arg b_{n}$ or $a_{n}=c b_{n}(0<c<1)$ and that $f$ is a meromorphic solution of (1.4). Set $k(z)=L(f)-\psi$. By $\sigma(\psi)<\infty$ and Lemma 2.8, we have $\sigma(k)=\infty$. Without loss of generality, we assume that $d_{2} \not \equiv 0$. Indeed, the remaining cases can be obtained by similar discussion. Substituting $L(f)=k(z)+\psi$ into (2.30), we have

$$
f=\frac{1}{h}\left(\alpha_{1}\left(k^{\prime}+\psi^{\prime}-\left(d_{2} F\right)^{\prime}-\alpha_{1} F\right)-\beta_{1}\left(k+\psi-d_{2} F\right)\right)=\frac{1}{h}\left(\alpha_{1} k^{\prime}-\beta_{1} k\right)+\phi,
$$

where

$$
\phi(z)=\frac{\alpha_{1}}{h}\left(\psi^{\prime}-\left(d_{2} F\right)^{\prime}-\alpha_{1} F\right)-\frac{\beta_{1}}{h}\left(\psi-d_{2} F\right)
$$

is a meromorphic function of finite order. Then substituting (4.1) into (1.4), we have

$$
\frac{\alpha_{1}}{h} k^{\prime \prime \prime}+\phi_{2} k^{\prime \prime}+\phi_{1} k^{\prime}+\phi_{0} k=F-\left(\psi^{\prime \prime}+A_{1} e^{P} \psi^{\prime}+A_{0} e^{Q} \psi\right)
$$

where $\phi_{j}(j=0,1,2)$ are meromorphic functions formed by $\alpha_{1} / h, \beta / h, \phi$, and their derivatives. If $F-\left(\psi^{\prime \prime}+A_{1} e^{P} \psi^{\prime}+A_{0} e^{Q} \psi\right) \equiv 0$, then by Theorem 1.2, we have $\sigma(\psi)=\infty$. This is impossible, and hence $F-\left(\psi^{\prime \prime}+A_{1} e^{P} \psi^{\prime}+A_{0} e^{Q} \psi\right) \not \equiv 0$. Thus, by $h \not \equiv 0, \alpha_{1} \not \equiv 0$, (4.3), and Lemma 2.4 , we get $\lambda(k)=\bar{\lambda}(k)=\sigma(k)=\infty$.

\section{Acknowledgments}

The author would like to thank the referees for their valuable suggestions and comments. This research was supported by NNSF of China (no. 11001057), NSF of Jiangsu Province (BK2010234), Project of Qinglan of Jiangsu Province.

\section{References}

[1] W. K. Hayman, Meromorphic Functions, Oxford Mathematical Monographs, Clarendon Press, Oxford, UK, 1964.

[2] E. Hille, Ordinary Differential Functions, Clarendon Press, Oxford, UK, 1964.

[3] Z. Chen, "The growth of solutions of $f^{\prime \prime}+e^{-z} f \prime+Q(z) f=0$ where the order $\sigma(Q)=1$," Science in China A, vol. 45, no. 3, pp. 290-300, 2002.

[4] G. G. Gundersen, "On the question of whether $f^{\prime \prime}+e^{-z} f^{\prime}+B(z) f=0$ can admit a solution $f \not \equiv 0$ of finite order," Proceedings of the Royal Society of Edinburgh A, vol. 102, no. 1-2, pp. 9-17, 1986.

[5] Z. X. Chen and K. H. Shon, "On the growth and fixed points of solutions of second order differential equations with meromorphic coefficients," Acta Mathematica Sinica (English Series), vol. 21, no. 4, pp. 753-764, 2005.

[6] J. Wang and I. Laine, "Growth of solutions of second order linear differential equations," Journal of Mathematical Analysis and Applications, vol. 342, no. 1, pp. 39-51, 2008.

[7] B. Belaïdi and A. El Farissi, "Relation between differential polynomials and small functions," Kyoto Journal of Mathematics, vol. 50, no. 2, pp. 453-468, 2010.

[8] J. Wang and I. Laine, "Growth of solutions of nonhomogeneous linear differential equations," Abstract and Applied Analysis, vol. 2009, Article ID 363927, 11 pages, 2009. 
[9] Z. X. Chen, "The fixed points and hyper order of solutions of second order complex differential equations," Acta Mathematica Scientia A, vol. 20, no. 3, pp. 425-432, 2000.

[10] J. Wang and W. R. Lü, "The fixed points and hyper-order of solutions of second order linear differential equations with meromorphic coefficients," Acta Mathematicae Applicatae Sinica, vol. 27, no. 1, pp. 72-80, 2004.

[11] I. Laine and J. Rieppo, "Differential polynomials generated by linear differential equations," Complex Variables. Theory and Application, vol. 49, no. 12, pp. 897-911, 2004.

[12] M.-S. Liu and X.-M. Zhang, "Fixed points of meromorphic solutions of higher order linear differential equations," Annales Academix Scientiarium Fennicæ. Mathematica, vol. 31, no. 1, pp. 191-211, 2006.

[13] Z. X. Chen and K. H. Shon, "The relation between solutions of a class of second-order differential equations with functions of small growth," Chinese Annals of Mathematics A, vol. 27, no. 4, pp. 431$442,2006$.

[14] G. G. Gundersen, "Estimates for the logarithmic derivative of a meromorphic function, plus similar estimates," Journal of the London Mathematical Society. Second Series, vol. 37, no. 1, pp. 88-104, 1988.

[15] A. I. Markushevich, Theory of Functions of a Complex Variable. Vol. II, Prentice-Hall, Englewood Cliffs, NJ, USA, 1965.

[16] Z. X. Chen, "Zeros of meromorphic solutions of higher order linear differential equations," Analysis, vol. 14, no. 4, pp. 425-438, 1994.

[17] C.-C. Yang and H.-X. Yi, Uniqueness Theory of Meromorphic Functions, vol. 557 of Mathematics and Its Applications, Kluwer Academic Publishers, Dordrecht, The Netherlands, 2003. 


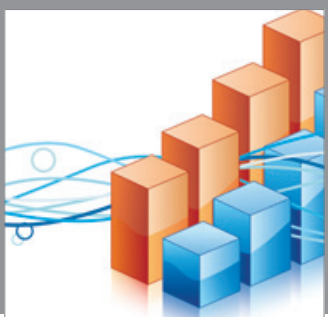

Advances in

Operations Research

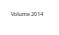

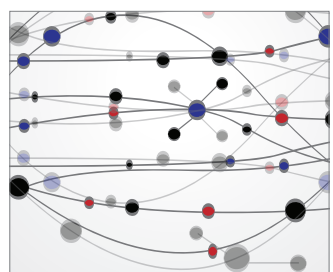

\section{The Scientific} World Journal
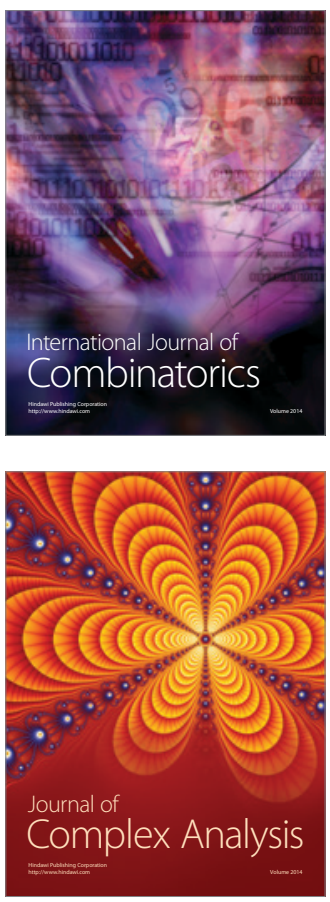

International Journal of

Mathematics and

Mathematical

Sciences
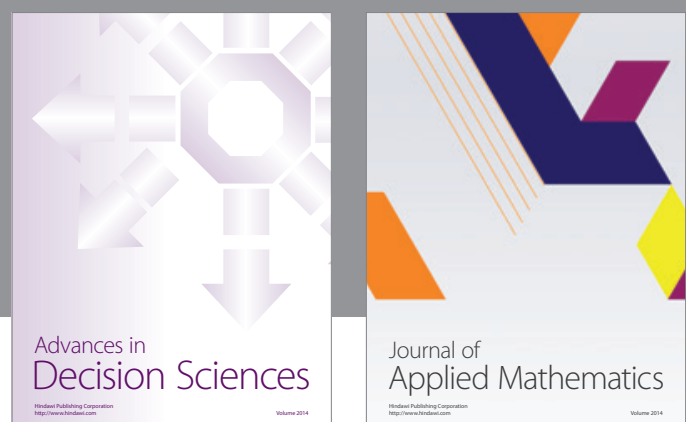

Journal of

Applied Mathematics
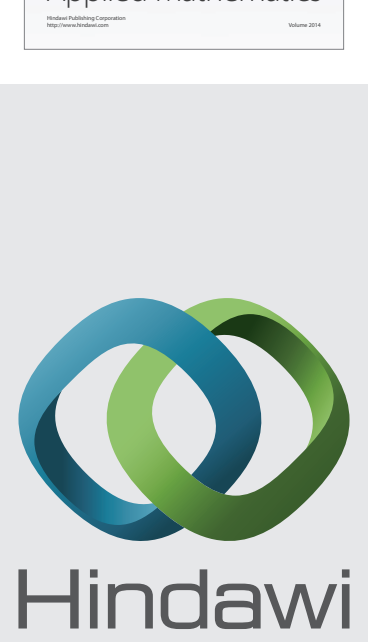

Submit your manuscripts at http://www.hindawi.com
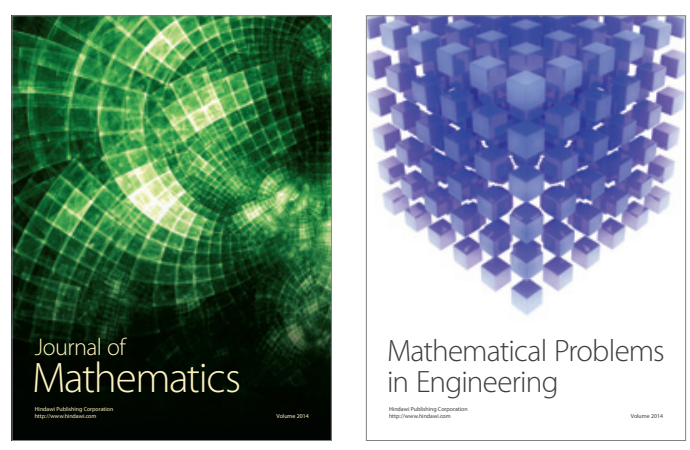

Mathematical Problems in Engineering
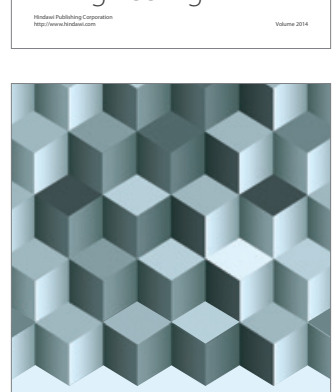

Journal of

Function Spaces
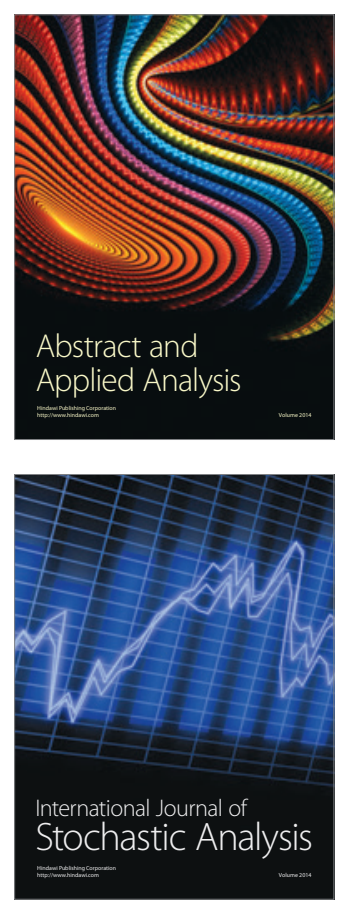

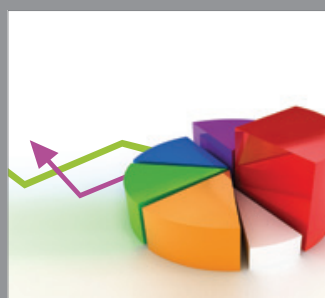

ournal of

Probability and Statistics

Promensencen
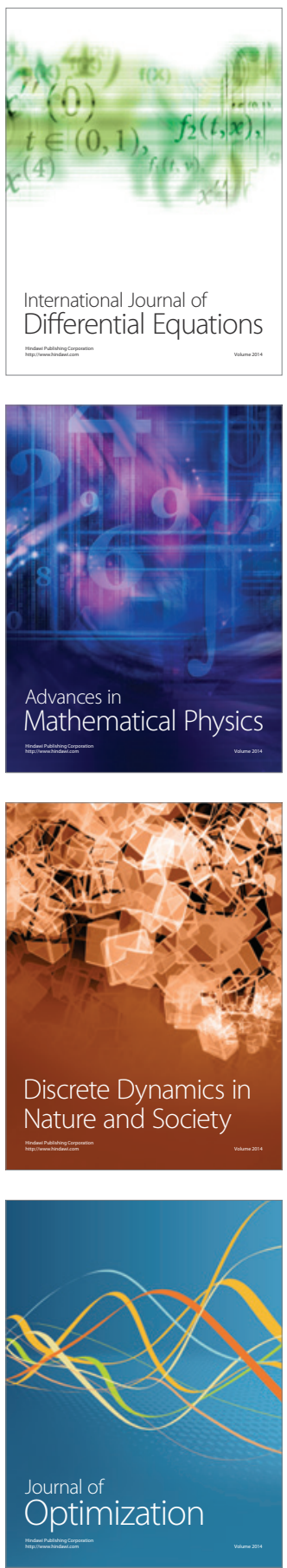